\title{
Regularity of the Density of States in the Anderson Model on a Strip for Potentials with Singular Continuous Distributions
}

\author{
Abel Klein, ${ }^{1}$ Jean Lacroix, ${ }^{1,2}$ and Athanasios Speis ${ }^{1,3}$
}

Received June 6, 1989

\begin{abstract}
We derive regularity properties for the density of states in the Anderson model on a one-dimensional strip for potentials with singular continuous distributions. For example, if the characteristic function is infinitely differentiable with bounded derivatives and together with all its derivatives goes to zero at infinity, we show that the density of states is infinitely differentiable.
\end{abstract}

KEY WORDS: Anderson model; density of states; Anderson model on a strip.

\section{INTRODUCTION}

To describe the motion of a quantum particle on a disordered crystal, Anderson ${ }^{(1)}$ introduced a model in which the particle, an electron, is assumed to interact only with the impurities in the crystal which produce a potential varying stochastically from site to site.

The Anderson model is given by the random Schrödinger operator

$$
H=-\frac{1}{2} \Delta+V
$$

acting on $l^{2}\left(\mathbf{Z}^{d}\right)$, where $\Delta$ is the finite-difference Laplacian and the values $V(x)$ of the potential at the lattice site $x$ are taken to be independent identically distributed random variables.

\footnotetext{
${ }^{1}$ Department of Mathematics, University of California, Irvine, California 92717.

${ }^{2}$ Permanent address: Département de Mathématiques, Université de Paris XIII, F-93430 Villetaneuse, France.

${ }^{3}$ Present address: Department of Mathematics, University of Michigan, Ann Arbor, Michigan 48109-1092.
} 
In the study of such random Hamiltonians one is usually not interested in the study of properties of $H$ for a fixed potential $V$, but only in properties that hold for typical $V$. For example, it is a consequence of ergodicity that the spectrum of the Hamiltonian $H$ is given by

$$
\sigma(H)=\sigma\left(-\frac{1}{2} A\right)+\operatorname{supp} \mu
$$

with probability one, ${ }^{(7,31)}$ where $\mu$ is the common probability distribution of the potential at a single site. The spectrum of $H$ can be decomposed into pure point spectrum, absolutely continuous spectrum, and singular continuous spectrum. This decomposition is independent of the potential with probability one. ${ }^{(31)}$

An important quantity in disordered systems is the density of states, which measures, in some sense, "how many states" corresponds to energies below a certain level.

The integrated density of states $N(E)$ is defined by

$$
N(E)=\lim _{A \rightarrow \mathbf{Z}^{d}} \frac{1}{|A|} \#\left\{\text { eigenvalues of } H_{A} \leqslant E\right\}
$$

where $A$ is a cube centered at the origin and $H_{A}$ denotes the operator $H$ restricted to $l^{2}(\Lambda)$ with Dirichlet boundary conditions.

It is a consequence of the ergodic theorem that for almost every potential the limit exists for all $E$ and is independent of the potential. ${ }^{(2-6)} N(E)$ is always a continuous function ${ }^{(7,8)}$ and under some mild conditions is logHolder continuous. ${ }^{(9)}$

It is known that without further restrictions on $\mu$ one should not expect too much more regularity. ${ }^{(11,12)}$ In one dimension under very general conditions $N(E)$ is always Hölder continuous on compact intervals ${ }^{(10,11)}$ and under some minimal regularity assumptions on $\mu$ it is always differentiable, even infinitely differentiable. ${ }^{(12-15)}$

In more than one dimension little is known about the differentiability of $N(E){ }^{(16-18)}$ Under mild regularity conditions which include the uniform distribution it follows that $N(E)$ is differentiable at high disorder ${ }^{(19)}$ and under some strong assumptions on $\mu$ (analytic density or exponential boundedness of its Fourier transform) it has been shown to be analytic at low energy or high disorder. ${ }^{(2,19,20)}$

In this paper we study the regularity of $N(E)$ for the Anderson model on a strip. This had previously been done by Klein and Speis ${ }^{(21)}$ under certain regularity assumptions on $\mu$ which required $\mu$ to be absolutely continuous (e.g., the uniform distribution). Their approach, following ref. 13, was to use the supersymmetric replica trick to rewrite the averaged Green's function as a two-point function of a supersymmetric field theory, 
which was then studied by a supersymmetric transfer matrix. The problem was reduced to the study of a certain eigenvector problem. Existence was not hard to establish, but the uniqueness followed from the uniqueness of a certain probability measure on the symplectic group which is invariant under an action associated with the random Schrödinger operator on the strip. Klein and Speis ${ }^{(21)}$ proved that the eigenvector was always the restriction of the Fourier transform of that invariant measure to a certain cone; to do so, they used an old result of Whitney ${ }^{(32)}$ to extend the eigenvector, originally defined on the cone of positive-semidefinite matrices, to the space of all symmetric matrices. This extension required a hypothesis on $\mu$ that excluded singular distributions (Ref. 21, Theorem IV.2.7).

In this paper we prove directly the uniqueness of the eigenvector under very weak hypothesis on $\mu$ (see Theorem 4.11); in particular, our conditions allow for singular continuous distributions.

This paper is organized as follows: In Section 2 we introduce the Anderson model on a strip and state our results; Section 3 is devoted to the development of the supersymmetric Hilbert spaces used in ref. 21 as well as to the introduction of some new ones which will be needed in the sequel. The supersymmetric transfer matrix and the eigenvalue problem are studied in Section 4. Finally, in Section 5 we derive the regularity of the density of states from the above-mentioned eigenvector as in ref. 21 .

\section{Statement of Results}

Let $\mathscr{D}_{m}$ be the one-dimensional lattice strip of width $m$, i.e., $\mathscr{D}_{m}=$ $\mathbf{Z} \times\{1, \ldots, m\}$, where $\mathbf{Z}$ is the set of all integers, $\mathbf{Z}^{+}$is the set of all nonnegative integers, and $m \in \mathbf{Z}^{+} \backslash\{0\}$. Let $\mathscr{D}^{1}=\{(1,0),(-1,0)\}$ and $\mathscr{D}^{2}=$ $\{(0,1),(0,-1)\}$.

The Anderson model ${ }^{(1)}$ on $\mathscr{D}_{m}$ is given by the random Hamiltonian $H=H_{0}+V$ on $l^{2}\left(\mathscr{D}_{m}\right)$, where

$$
\left(H_{0} u\right)(x)=\sum_{y \in \mathscr{I}_{m}}\left(H_{0}\right)_{x y} u(y)
$$

with

$$
\begin{aligned}
\left(H_{0}\right)_{x y} & = \begin{cases}-1 / 2 & \text { if } x-y \in \mathscr{D}^{1} \\
0 & \text { otherwise }\end{cases} \\
(V u)(x) & =\sum_{y \in \mathscr{O}_{m}} V_{x y} u(y)
\end{aligned}
$$


with

$$
V_{x y}= \begin{cases}-1 / 2 & \text { if } x-y \in \mathscr{D}^{2} \\ V(x) & \text { if } x=y \\ 0 & \text { otherwise }\end{cases}
$$

and $\{V(x)\}_{x \in \mathscr{Q}_{m}}$ are i.i.d. real random variables with a common probability distribution $\mu$ whose characteristic function will be denoted by $h$. We will always assume that

$$
\int \log (1+|v|) d \mu(v)<+\infty
$$

Let $l_{1} \leqslant l_{2} \in \mathbf{Z}$ and let $A_{\left[l_{1}, l_{2}\right]}$ be the box $\left[l_{1}, l_{2}\right] \times\{1,2, \ldots, m\}$ in $\mathscr{D}_{m}$. We will denote by $H_{\left[l_{1}, l_{2}\right]}$ the operator $H$ restricted to $l^{2}\left(A_{\left[l_{1}, l_{2}\right]}\right)$ with boundary condition $u(x)=0$ for all $x \notin \Lambda_{\left[l_{1}, i_{2}\right]}$.

Let $l \in \mathbf{Z}^{+}$; we will denote by $A_{l}$ the set $A_{[-l, l]}$ and by $H_{l}$ the operator $H_{[-l, l]}$. We will also denote by $\left|A_{l}\right|$ the number of points in $A_{l}$.

The integrated density of states $N(E), E \in \mathbf{R}$, is defined by

$$
\left.N(E)=\lim _{l \rightarrow \infty}\left|\Lambda_{l}\right|^{-1} \# \text { eigenvalues of } H_{l} \leqslant E\right\}
$$

As in $\mathbf{Z}^{d}$, for almost every potential the limit exists for all $E$ and is a continuous function of $E^{(2-9)}$

On a one-dimensional strip, Klein et al. ${ }^{(23)}$ showed that if $\mu$ is not concentrated in a single point and $\int|v|^{\alpha} d \mu(v)<+\infty$ for some $\alpha>0$, then $N(E)$ is locally Hölder continuous. Under the hypothesis that $h$ is of class $C^{d}$ on $[0,+\infty)$ with $4 d \geqslant 3 m^{2}-m+4$ and $h^{(j)}(t)=O\left(1+|t|^{-\alpha}\right)$ for some $\alpha>1 / 2$ and all $j=0,1, \ldots, d$, Klein and Speis ${ }^{(21)}$ showed that $N(E)$ is of class $C^{[(d+1) / 2]}$. They also proved that if $h$ is of class $C^{\left(3 m^{2}-m+4\right) / 4}$ on $[0,+\infty)$ with $h^{(j)}(t)=O\left(1+|t|^{-\alpha}\right)$ for some $\alpha>1 / 2$ and all $j=0, \quad 1, \ldots$, $\frac{1}{4}\left(3 m^{2}-m+4\right)$ and $h^{(j)}(t)=O\left(e^{-b|t|}\right)$ for $j=0,1$ and some $b>0$, then $N(E)$ has an analytic extension to a strip about the real axis. Recently Glaffig $^{(22)}$ proved that if the potential on the top line consists of i.i.d. random variables with compactly supported distributions $F(x) d x$ such that $F \in L_{\alpha}^{1}=\left\{f \in L^{1}(\mathbf{R})\right.$; there exists $g \in L^{1}(\mathbf{R})$ such that $\left.\hat{g}(t)=\left(1+t^{2}\right)^{\alpha / 2} \hat{f}(t)\right\}$, with $\alpha>0$, and on the rest of the strip consists of i.i.d, Bernoulli random variables with possible values 0 and $b$, independent of the ones on the top line, then then exists $b_{0}>0$ which depends on $F$ such that if $|b|<b_{0}$, then $N(E)$ is of class $C^{\infty}$.

In this paper we prove the same type of regularity as in ref. 21 under much weaker hypotheses, similar to the ones Klein and Speis used in ref. 15 . We now state our results. 
Theorem 2.1. Let $d \in \mathbf{Z}^{+} \backslash\{0\}$. Let $h$ be bounded and of class $C^{d}$ with bounded derivatives on $(0,+\infty)$. If $h^{(j)}(r) \rightarrow 0$ as $r \rightarrow+\infty$ for all $j=0,1, \ldots, d$, then $N(E)$ is of class $C^{[(d+1) / 2]}$.

Theorem 2.2. Let $h$ be differentiable on $(0,+\infty)$ and let

$$
\left|h^{(j)}(r)\right| \leqslant M e^{-x r}
$$

for some $M<+\infty, \alpha>0$, all $r>0$ and $j=0,1$. Then $N(E)$ has an analytic extension to $\left\{z \in \mathbf{C}:|\operatorname{Im} z|<b_{1}\right\}$ for some $b_{1}>0$.

\section{SUPERSYMMETRIES AND SUPERSYMMETRIC SPACES}

\subsection{Functions Defined on Superspaces}

Definition 3.1. Let $n, m$ be two positive integers. Let $A\left(\mathbf{R}^{2 n m}\right)$ denote the Grassman algebra over $\mathbf{R}^{2 n m}$ and let $\Lambda^{1}\left(\mathbf{R}^{2 n m}\right)$ be the vector space of 1 -forms in $\Lambda\left(\mathbf{R}^{2 n m}\right)$.

The superspace $\mathscr{L}_{n, m}$ is defined to be the set of all $n$-tuples $\Phi=$ $\left(\Phi_{1}, \ldots, \Phi_{n}\right)$ where

$$
\begin{gathered}
\Phi_{i}=\left(\varphi_{i}, \bar{\psi}_{i}, \psi_{i}\right), \quad i=1, \ldots, n \\
\varphi_{i} \in \mathbf{R}^{2}, \quad i=1, \ldots, n
\end{gathered}
$$

and $\left\{\bar{\psi}_{1}, \psi_{i}\right\}_{i}=1, \ldots, n$ is a family of 1 -forms with its nonzero elements forming a linearly independent set in $A^{1}\left(\mathbf{R}^{2 n m}\right)$.

Definition 3.2. Let $I$ be a finite index set, let $n \in \mathbf{Z}$, and let $|\boldsymbol{I}|$ denote the number of elements of $I$.

We define $\mathscr{L}_{n}^{I}$ to be the set of all functions $\Phi: I \rightarrow \mathscr{L}_{n,|I|}$ of the form $\Phi(x)=\left(\Phi_{1}(x), \ldots, \Phi_{n}(x)\right), x \in I$, where $\Phi_{1}, \ldots, \Phi_{n}$ are functions from $I$ to $\mathscr{L}_{1,|Y|}$ with $\Phi_{i}(x)=\left(\varphi_{i}(x), \bar{\psi}_{i}(x), \psi_{i}(x)\right) \in \mathscr{L}_{1,|,|}$ for all $x \in I$ and for all $i \in\{1, \ldots, n\} ; \varphi_{1}, \ldots, \varphi_{n}$ are functions from $I$ to $\mathbf{R}^{2}$, and $\bar{\psi}_{1}, \psi_{1}, \ldots, \bar{\psi}_{n}, \psi_{n}$ are functions from $I$ to $A^{1}\left(\mathbf{R}^{2 n|I|}\right)$, such that $\left\{\psi_{i}(x), \psi_{i}(x)\right\}_{x \in I, i=1, \ldots, n}$ is a family of 1 -forms with its nonzero elements forming a linearly independent set in $A^{1}\left(\mathbf{R}^{2 n|l|}\right)$.

We will also be using the notation $\Phi=(\varphi, \psi, \psi)$, where $\varphi$ is a function from $I$ to $\mathbf{R}^{2 n}$ with $\varphi=\left(\varphi_{1}, \ldots, \varphi_{n}\right)$ and $\psi, \bar{\psi}$ are functions from $I$ to $A^{1}\left(\mathbf{R}^{2 n|f|}\right)$ with $\psi=\left(\psi_{1}, \ldots, \psi_{n}\right), \bar{\psi}=\left(\bar{\psi}_{1}, \ldots, \bar{\psi}_{n}\right)$.

Definition 3.3. Let $n \in \mathbf{Z}$, let $I$ and $I^{\prime}$ be two finite index sets with $I \supset I^{\prime}$. Let $\tilde{\mathscr{L}}_{n}^{I^{\prime}}=\left\{\Phi \in \mathscr{L}_{n}^{I^{\prime}}: \Phi(x)=0 \forall x \notin I^{\prime}\right\}$. We say that $\tilde{\mathscr{L}}_{n}^{I^{\prime}}$ is the copy of $\mathscr{L}_{n}^{I^{\prime}}$ in $\mathscr{L}_{n}^{l}$.

Let $\left\{\mathscr{L}_{n}^{I_{i}}\right\}_{i=1, \ldots, m}$ be a family of superspaces with $\left\{I_{i}\right\}_{i=1, \ldots, m}$ a family 
of index sets not necessary distinct. We define $X_{i=1}^{m} \mathscr{L}_{n}^{l_{i}}$ to be the usual Cartesian product between the $\mathscr{L}_{n}^{I_{i}}$.

Let $I$ be the disjoint union of $I_{i}$ and let $\tilde{\mathscr{L}}_{n}^{I_{i}}$ be the copy of $\mathscr{L}_{n}^{I_{i}}$ in $\mathscr{L}_{n}^{I}$, $\forall i=1, \ldots, m$; then $X_{i=1}^{m} \tilde{\mathscr{L}}_{n}^{I_{i}}$ can be identified in a canonical way with $\mathscr{L}_{n}^{I}$.

Since for the purposes of this paper the specific linear dimensions of the Grassmann algebras in Definitions 3.1 and 3.2 are not important as long as we have enough linearly independent 1-forms, we will not be distinguishing between different copies of a superspace. Consequently we will always assume that $X_{i=1}^{m} \mathscr{L}_{n}^{I_{i}}$ is a particular case of a superspace as it is defined in Definition 3,2.

For the sake of clarity of our notation we will always be assuming that all the index sets that appear are equipped with a fixed linear order $(\leqslant)$. Let $I$ be an index set; for $x, y \in I$ we will write $x<y$ if and only if $x \leqslant y$ and $x \neq y$.

Notations. Let $I$ be a finite index set and let $2^{I}$ be the set of all the subsets of $I$. Set $S_{I}=2^{I} \times 2^{I}$ and let $\Phi=(\varphi, \psi, \psi)$ be an element of $\mathscr{L}_{n}^{I}$ for some $n \in \mathbf{Z}^{+} \backslash\{0\}$. For every $a$ in $S_{I}^{n}$ we assign to $\Phi$ an element $\psi_{a}$ of $\Lambda\left(\mathbf{R}^{2 n|I|}\right)$ defined by the formula $\psi_{a}=\prod_{i=1}^{n} \psi_{i}\left(\bar{a}_{i}, a_{i}\right)$ where

$$
\begin{array}{rlrl}
a & =\left(\left(\bar{a}_{1}, a_{1}\right), \ldots,\left(\bar{a}_{n}, a_{n}\right)\right) \quad \text { with } \quad \bar{a}_{i}, a_{i} \in 2^{I}, \quad \forall i=1, \ldots, n \\
\psi(\bar{D}, D) & =\prod_{j=1}^{|\bar{D}|} \bar{\psi}_{i}\left(\bar{x}_{j}\right) \prod_{j=1}^{|D|} \psi_{i}\left(x_{j}\right), \quad(\bar{D}, D) \in S_{I} \\
\bar{x}_{1} & <\cdots<\bar{x}_{|\bar{D}|} \in \bar{D} \quad \text { and } & x_{1}<\cdots<x_{|D|} \in D
\end{array}
$$

and $|\bar{D}|$ and $|D|$ are the number of elements in $D$ and $D$, respectively.

Let $a \in S_{I}^{n}$ and let $\sigma$ be a permutation in $n$ symbols. Let $\sigma(a)$ be the element of $S_{I}^{n}$ which is defined by

$$
\sigma(a)=\left(\left(\bar{a}_{\sigma(1)}, a_{\sigma(1)}\right), \ldots,\left(\bar{a}_{\sigma(n)}, a_{\sigma(n)}\right)\right)
$$

where $a=\left(\left(\bar{a}_{1}, a_{1}\right), \ldots,\left(\bar{a}_{n}, a_{n}\right)\right)$. Let $x \in I$ and $p \in\{1, \ldots, n\}$; we define the following operations on elements of $S_{I}^{n}$ :

$$
\begin{aligned}
\bar{\Pi}_{p}^{x} a & =\left(\left(\bar{a}_{1}, a_{1}\right), \ldots,\left(\bar{a}_{p} \cup\{x\}, a_{p}\right), \ldots,\left(\bar{a}_{n}, a_{n}\right)\right) \\
\Pi_{p}^{x} a & =\left(\left(\bar{a}_{1}, a_{1}\right), \ldots,\left(\bar{a}_{p}, a_{p} \cup\{x\}\right), \ldots,\left(\bar{a}_{n}, a_{n}\right)\right) \\
\Omega_{p}^{x} a & =\left(\left(\bar{a}_{1}, a_{1}\right), \ldots,\left(\bar{a}_{p}, a_{p} \backslash\{x\}\right), \ldots,\left(\bar{a}_{n}, a_{n}\right)\right) \\
\bar{\Omega}_{p}^{s} a & =\left(\left(\bar{a}_{1}, a_{1}\right), \ldots,\left(\bar{a}_{p} \backslash\{x\}, a_{p}\right), \ldots,\left(\bar{a}_{n}, a_{n}\right)\right) \\
\Omega_{p} a & =\left(\left(\bar{a}_{1}, a_{1}\right), \ldots,(\varnothing, \varnothing), \ldots,\left(\bar{a}_{n}, a_{n}\right)\right) \\
\Pi_{p} a & =\left(\left(\bar{a}_{1}, a_{1}\right), \ldots,(I, I), \ldots,\left(\bar{a}_{n}, a_{n}\right)\right)
\end{aligned}
$$

where $\varnothing$ is the empty set, and all changes were done in the $p$ th position. 
We will be denoting $((\varnothing, \varnothing), \ldots,(\varnothing, \varnothing))$ by $a_{\varnothing}$ and $((I, I), \ldots,(I, I))$ by $a_{i}$. We will also be using the notation

$$
\sum_{p=1}^{n}\left|\bar{a}_{p}\right|+\left|a_{p}\right|=|a|
$$

Definition 3.4. Let $I, n$ be as in Definition 3.2. A superfunction $F$ defined on $\mathscr{L}_{n}^{I}$ is understood to be a function $F: \mathscr{L}_{n}^{I} \rightarrow A\left(\mathbf{R}^{2 n|I|}\right)$ of the form

$$
F(\Phi)=\sum_{a \in S_{I}^{n}} F_{a}(\varphi) \psi_{a}
$$

where $\Phi=(\varphi, \bar{\psi}, \psi) \in \mathscr{L}_{n}^{I}$, and $\left\{F_{a}\right\}_{a \in S_{t}^{n}}$ is a family of complex-valued functions defined on $\mathbf{R}^{2 n|\ell|}$.

The notation

$$
F\left(\Phi_{1}, \ldots, \Phi_{n}\right)=\sum_{a \in S_{l}^{n}} F_{a}\left(\varphi_{1}, \ldots, \varphi_{n}\right) \psi_{a}
$$

will also be used.

We will say that a superfunction $F$ is in $C^{k}\left(\mathscr{L}_{n}^{I}\right)$ if and only if $F_{a}$ is of class $C^{k}$ on $\mathbf{R}^{2 n|I|} \forall a \in S_{I}^{n}$. We will also say that $F$ is in $C_{c}^{k}\left(\mathscr{L}_{n}^{I}\right)$ if and only if $F_{a}$ is of class $C^{k}$ and has compact support in $\mathbf{R}^{2 n|I|} \forall a \in S_{I}^{n}$.

Definition 3.5. (Also see refs. 21 and 25-27.) Let $I, n$ be as in Definition 3.2 and let $a, a^{\prime} \in S_{I}^{n}$. We will say that $a^{\prime} \supset a$ if and only if $\bar{a}_{p} \subset \bar{a}_{p}^{\prime}, a_{p} \subset a_{p}^{\prime} \forall p \in\{1, \ldots, n\}$.

Let $I^{\prime}$ be a subset of $I$ and $M$ a subset of $\{1, \ldots, n\}$. We define the notation of superintegration through the formula

$$
\begin{aligned}
(-1)^{C_{a}} \int F(\Phi) \prod_{\substack{i \in M \\
x \in I^{\prime}}} d^{2} \varphi_{i}(x) d \psi_{a} \\
=(-1)^{C_{a}} \int F(\Phi) d \psi_{a} \prod_{\substack{i \in M \\
x \in I^{\prime}}} d^{2} \varphi_{i}(x) \\
=(-1)^{|a| / 2} \sum_{a^{\prime} \supset a}(-1)^{C_{a^{\prime} \backslash a}} \psi_{a^{\prime} \backslash a} \\
\quad \times \int(-1)^{C_{a^{\prime}}} F_{a^{\prime}}(\varphi) \prod_{\substack{i \in M \\
x \in I^{\prime}}} d^{2} \varphi(x)
\end{aligned}
$$

where

$$
a^{\prime} \backslash a=\left(\left(\bar{a}_{1}^{\prime} \backslash \bar{a}_{1}, a_{1}^{\prime} \backslash a_{1}\right), \ldots\right)
$$


$F$ is a superfunction defined over $\mathscr{L}_{n}^{I}$ such that

$$
\int\left|F_{a}(\varphi)\right| \prod_{\substack{i \in M \\ x \in I^{\prime}}} d^{2} \varphi_{i}(x)<\infty \quad \text { for all } a \in S_{I}^{n}
$$

and

$$
C_{a}=\sum_{p=1}^{n} \frac{\left|\bar{a}_{p}\right|\left(\left|\bar{a}_{p}\right|+1\right)}{2}, \quad a \in S_{I}^{n}
$$

If $k \leqslant n,\left\{i_{1}, \ldots, i_{k}\right\} \subset\{1, \ldots, n\}$, and $a=\Pi_{i_{k}} \ldots \Pi_{i_{1}} a_{\varnothing}$ we will be using the notation $d \Phi_{i_{1}} \cdots d \Phi_{i_{k}}$ for

$$
(-1)^{C_{a}} \frac{1}{\pi^{k n}} \prod_{x \in I} \prod_{i=1}^{k} d^{2} \varphi_{i}(x) d \psi_{a}
$$

If $k=n$, the notation $d \Phi$ will be used instead of $d \Phi_{1} \cdots d \Phi_{n}$.

We will say that a superfunction $F$ defined on $\mathscr{L}_{n}^{I}$ belongs to $L^{p}\left(\mathscr{L}_{n}^{I}\right)$ if and only if $F_{a} \in L^{p}\left(\mathbf{R}^{2 n|I|}\right) \forall a \in S_{I}^{n}$.

\subsection{Supersymmetries and Supersymmetric Functions}

Let $I, n$ be as in Section 3.1.

Definition 3.6. (See also refs. 13, 19, and 21.) Let $\Phi, \Phi^{\prime} \in \mathscr{L}_{n,|I|}$. We define a dot product between $\Phi$ and $\Phi^{\prime}$ through the formula $\Phi \cdot \Phi^{\prime}=$ $\sum_{i=1}^{n} \Phi_{i} \cdot \Phi_{i}^{\prime}$, where

$$
\Phi_{i} \cdot \Phi_{i}^{\prime}=\varphi_{i} \cdot \varphi_{i}^{\prime}+\frac{1}{2}\left(\bar{\psi}_{i} \psi_{i}^{\prime}+\bar{\psi}_{i}^{\prime} \psi_{i}\right), \quad i=1, \ldots, n
$$

Supersymmetric transformations are "rotations" of the superspace $\mathscr{L}_{n,|i|}$ (see ref. 27) which leave the above dot product invariant. More specifically, in addition to the usual rotations of $\mathbf{R}^{2 n}$ and symplectic transformations of $A\left(\mathbf{R}^{2 n|f|}\right)$ they include transformations of the type $\mathscr{L}_{n,|I|} \ni \Phi \rightarrow \mathscr{C}_{\xi, b, b}^{p}(\Phi)$, where

$$
\begin{aligned}
\mathscr{C}_{\xi, b, b}^{p}\left(\Phi_{1}, \ldots, \Phi_{n}\right) & =\left(\Phi_{1}, \ldots, \mathscr{C}_{\xi, b, b}\left(\Phi_{p}\right), \ldots, \Phi_{n}\right) \\
\mathscr{C}_{\xi, b, b}\left(\Phi_{p}\right) & =\left(\varphi_{p}+2 b \xi \psi_{p}+2 b \xi \psi_{p}, \bar{\psi}_{p}-4 \bar{b} \varphi_{p} \xi, \psi_{p}+4 b \varphi_{p} \xi\right)
\end{aligned}
$$

$\xi$ is a 1 -form in $A^{1}\left(\mathbf{R}^{2 n|I|+1}\right)$ such that $\xi(\omega)=0$ for all $\omega \in \mathbf{R}^{2 n|l|}$, and $b$, $\bar{b} \in \mathbf{R}^{2}$.

Definition 3.7. A supersymmetry $\vec{u}$ of $\mathscr{L}_{n}^{l}$ is a transformation of the type $\mathscr{L}_{n}^{I} \ni \Phi \rightarrow \vec{u}(\Phi)$, where $[\vec{u} \Phi](x)=u(\Phi(x)) \forall x \in I$ and $u$ is a super- 
symmetric transformation of $\mathscr{L}_{n,|I|}$. The action of supersymmetries on superfunctions defined on $\mathscr{L}_{n}^{I}$ will now be defined. If $u$ is a usual rotation or a pure symplectic transformation, the action of $\vec{u}$ is obvious. If $u$ is of the type $\mathscr{C}_{\xi, b, 5}^{p}$, the action of $\dot{u}$ on a superfunction $F$ is defined by

$$
\begin{gathered}
(\vec{u} F)(\Phi)=F(\vec{u} \Phi) \\
g\left(\varphi_{1}(x), \ldots, \varphi_{p}(x)+2 \bar{b} \xi \psi_{p}(x), \ldots, \varphi_{n}(x)\right) \\
=g\left(\varphi_{1}(x), \ldots, \varphi_{n}(x)\right)+2 \nabla_{\varphi_{p}(x)} g\left(\varphi_{1}(x), \ldots, \varphi_{n}(x)\right) \\
\cdot\left(\bar{b} \xi \psi_{p}(x)+b \xi \bar{\psi}_{p}(x)\right)
\end{gathered}
$$

where $g: \mathbf{R}^{2 n} \rightarrow \mathbf{C}$ is a function in $C^{1}\left(\mathbf{R}^{2 n}\right)$ and $\forall x \in I, \nabla_{\varphi_{p}(x)}$ is the usual gradient with respect to $\varphi_{p}(x) \in \mathbf{R}^{2}$ for all $x \in I$.

If $u$ is of the type $\mathscr{C}_{\tilde{\xi}, b, \zeta}^{p}$ and $F \in C^{1}\left(\mathscr{L}_{n}^{I}\right)$, then, following the notation of Section 3.1 and using (3.3), it is easy to show that $(\vec{u} F)(\Phi)=F(\vec{u} \Phi)=$ $F(\Phi)+F^{\prime}(\Phi) \xi$, where $F^{\prime} \in C\left(\mathscr{L}_{n}^{l}\right)$ and $\forall a \in S_{l}^{n}$

$$
\begin{aligned}
F_{a}^{\prime}(\varphi)= & \sum_{x \notin a_{p}}(-1)^{a_{p}(x)} 4 \varphi_{p}(x) \cdot b F_{\Pi_{p}^{c} c^{c}}(\varphi) \\
& +\sum_{x \in \bar{a}_{p}}(-1)^{\bar{a}_{p}(x)+1} 2 b \cdot \nabla_{\varphi_{p}(x)} F_{\bar{\Omega}_{p}^{x}}(\varphi) \\
& +\sum_{x \notin \bar{a}_{p}}(-1)^{\bar{a}_{p}(x)+1} 4 \varphi_{p}(x) \cdot \bar{b} F_{\pi_{p}^{x}}(\varphi) \\
& +\sum_{x \in a_{p}}(-1)^{a_{p}(x)+1} 2 \bar{b} \cdot \nabla_{\varphi_{p}(x)} F_{\Omega_{p}^{x}}(\varphi)
\end{aligned}
$$

where $a_{p}(x)=\#$ of 1 -forms in front of $\psi_{p}(x)$ in $\psi_{\Pi_{p}^{x}}$ and $\bar{a}_{p}(x)=\#$ of 1 -forms in front of $\bar{\psi}_{p}(x)$ in $\psi_{\Pi_{p}^{x}}^{x}$.

Definition 3.8. Let $n, I$ be as in Definition 3.2. A superfunction $F$ in $C\left(\mathscr{L}_{n}^{I}\right)$ is called supersymmetric if it is left invariant by all sypersymmetries of $\mathscr{L}_{n}^{I}$.

We will say that a superfunction $F$ defined over $\mathscr{L}_{n}^{\prime}$ is in $S C^{k}\left(\mathscr{L}_{n}^{\prime}\right)$ $\left[S C_{c}^{k}\left(\mathscr{L}_{n}^{l}\right)\right]$ if and only if $F$ is supersymmetric and $F$ is in $C^{k}\left(\mathscr{L}_{n}^{n}\right)$ $\left[C_{c}^{k}\left(\mathscr{L}_{n}^{I}\right)\right]$. We will also say that $F \in S \mathscr{S}\left(\mathscr{L}_{n}^{I}\right)$ if and only if $F$ is supersymmetric and $F_{a} \in \mathscr{S}\left(\mathbf{R}^{2 n|I|}\right) \forall a \in S_{I}^{n}$, where $\mathscr{P}\left(\mathbf{R}^{2 n|I|}\right)$ is the usual Schwartz space over $\mathbf{R}^{2 n|I|}$.

Proposition 3.9. Let $k, n$ be a positive integers such that $k \leqslant n$. Let $\vec{u}$ be a supersymmetric of $\mathscr{L}_{n}^{\prime}$ with the property $(\vec{u} \Phi)_{j}=\Phi_{j}, \forall j>k$ and $\forall \Phi \in \mathscr{L}_{n}^{\prime}$. Then for any $F \in C^{1}\left(\mathscr{L}_{n}^{I}\right) \cap L^{1}\left(\mathscr{L}_{n}^{I}\right)$

$$
\int(\vec{u} F)\left(\Phi_{1}, \ldots, \Phi_{n}\right) d \Phi_{1} \ldots d \Phi_{k}=\int F\left(\Phi_{1}, \ldots, \Phi_{n}\right) d \Phi_{1} \ldots d \Phi_{k}
$$


Proof. The only non-obvious case is when $u$ is of the type $\mathscr{C}_{\xi, b, 5}^{p}$, in which case the proof follows as in ref. 27, Proposition 4.2.

Notations. Let $M_{I}$ be the set of $|I| \times|I|$ real, symmetric matrices and let $M_{I}^{+}$be the set of all positive-semidefinite elements of $M_{I}$. Topologically, we identify $M_{I}$ with $\mathbf{R}^{1 / 2|I|(I I \mid+1)}$. Let $\varphi$ be a function from $I$ to $\mathbf{R}^{2 n}$; we associate to $\varphi$ an element $A_{\varphi}$ of $M_{I}$ defined by the formula $\left(A_{\varphi}\right)_{x, y}=$ $\varphi(x) \cdot \varphi(y), x \in I$. Let $\Phi=(\varphi, \bar{\psi}, \psi)$ be an element of $\mathscr{L}_{n}^{I}$; we shall make use of the supermatrix $A_{\Phi}$ defined by $A_{\Phi}=A_{\varphi}+A_{\psi, \psi}$, where

$$
\left(A_{\psi, \psi}\right)_{x y}=\frac{1}{2} \sum_{p=1}^{n} \bar{\psi}_{p}(x) \psi_{p}(y)+\bar{\psi}_{p}(y) \psi_{p}(x)
$$

Trivially $\left(A_{\Phi}\right)_{x, y}=\Phi(x) \cdot \Phi(y), x, y \in I$.

Let $e$ be an element of $M_{I}$ with its matrix elements being positive integers. We shall make use of the notations

$$
\begin{aligned}
\partial^{e} & =\prod_{\substack{x, y \in I \\
x \leqslant y}} \frac{\partial^{e_{x y}}}{\partial A_{x y}^{e_{x y}}} \\
A_{\Psi, \psi}^{e} & =\prod_{\substack{x, y \in I \\
x \leqslant y}}\left(A_{\Psi, \psi}\right)^{e_{x y}}
\end{aligned}
$$

We will say that $e$ is $I$-admissible of order $n$ if and only if $\left|e_{x y}\right| \leqslant n$, for all $x, y \in I$.

To every element $(\bar{D}, D)$ of $S_{I}$ with the property $|\bar{D}|=|D|=d$ we associate a $d \times d$ matrix $\mathcal{O}_{(\bar{D}, D)}$ defined by the formula

$$
\mathcal{O}_{(\bar{D}, D)}=\left[\begin{array}{llll}
\frac{\partial}{\partial A_{\bar{D}_{1}, D_{1}}} & \frac{1}{2} \frac{\partial}{\partial A_{\bar{D}_{1}, D_{2}}} & \cdots & \frac{1}{2} \frac{\partial}{\partial A_{\bar{D}_{1}, D_{d}}} \\
\frac{1}{2} \frac{\partial}{\partial A_{\bar{D}_{2}, D_{1}}} & \frac{\partial}{\partial A_{\bar{D}_{2}, D_{2}}} & \cdots & \frac{1}{2} \frac{\partial}{\partial A_{\bar{D}_{2}, D_{d}}} \\
\vdots & \vdots & & \\
\frac{1}{2} \frac{\partial}{\partial A_{\bar{D}_{d}, D_{1}}} & \frac{1}{2} \frac{\partial}{\partial A_{\bar{D}_{d}, D_{2}}} & \cdots & \frac{\partial}{\partial A_{\bar{D}_{d}, D_{d}}}
\end{array}\right]
$$

where $\partial / \partial A_{\bar{D}_{i, D_{j}}}$ is the partial derivative with respect to $A_{\bar{D}_{i, D_{j}}}$ and $\bar{D}_{1}<$ $\bar{D}_{2}<\cdots<\bar{D}_{d} \in \bar{D}, D_{1}<D_{2}<\cdots<D_{d} \in D$. We will be making use of the operators $\partial(\bar{D}, D)$ which acts on functions defined on $M_{I}^{+}$and they are defined by the formula

$$
\partial_{(\bar{D}, D)}=\operatorname{det}\left[\mathscr{U}_{(\bar{D}, D)}\right]
$$

if $|\bar{D}|=|D|$ and zero otherwise. 
Let $k \in \mathbf{Z}^{+}$. We will say that a complex-valued function $f$ defined over $M_{I}^{+}$is in $C_{k}\left(M_{I}^{+}\right)$if and only if it is of class $C^{\infty}$ in the interior of $M_{I}^{+}$ and $\partial_{a} f$ is continuous all the way up to the boundary for all $a$ in $S_{I}^{k}$, where

$$
\partial_{a}=\prod_{p=1}^{k}(-1)^{|\bar{a}|\left(\left|\bar{a}_{p}\right|+1\right) / 2} \partial_{\left(\bar{a}_{p}, a_{p}\right)}
$$

and $a=\left(\left(\bar{a}_{1}, a_{1}\right), \ldots,\left(\bar{a}_{k}, a_{k}\right)\right) \in S_{I}^{k}$.

To every element $f$ of $C_{k}\left(M_{I}^{+}\right)$we associate a supersymmetric function $L_{k}^{I}(f)$ which is defined by

$$
F(\Phi)=f\left(A_{\Phi}\right), \quad \Phi \in \mathscr{L}_{k}^{I}
$$

where $f\left(A_{\Phi}\right)$ is defined as usual through a formal Taylor expansion,

$$
f\left(A_{\Phi}\right)=\sum_{\substack{e \ell \text {-adm } \\ \text { of order } k}} \frac{1}{e !} \partial^{e} f\left(A_{\varphi}\right) A_{\psi, \psi}^{e}
$$

where

$$
e !=\prod_{\substack{x, y \in I \\ x \leqslant y}}\left(e_{x y}\right) !
$$

Remark 3.10. Let $f \in C_{k}\left(M_{I}^{+}\right)$. It is easy to see that

$$
L_{k}^{I}(f)(\Phi)=f\left(A_{\Phi}\right)=\sum_{a \in S_{I}^{k}} \partial_{a} f\left(A_{\varphi}\right) \psi_{a}
$$

and that $L_{k}^{I}$ maps $C_{k}\left(M_{I}^{+}\right)$into $S C^{0}\left(\mathscr{L}_{k}^{I}\right)$ for all $k \in \mathbf{Z}^{+} \backslash\{0\}$.

Definition 3.11. Let $k \in \mathbf{Z}^{+} \backslash\{0\}$; we will say that a complexvalued function $f$ defined on $M_{I}^{+}$is in $\mathscr{L}^{k}\left(M_{I}^{+}\right)$if and only if $f \in C_{k}\left(M_{I}^{+}\right)$ and $L_{k}^{I}(f) \in S \mathscr{P}\left(\mathscr{L}_{k}^{I}\right)$.

Theorem 3.12. If $n \in \mathbf{Z}^{+} \backslash\{0\}$ with $n \geqslant|I| / 2$, then the function

$$
L_{n}^{I}: \quad \mathscr{S}^{n}\left(M_{I}^{+}\right) \rightarrow S \mathscr{P}\left(\mathscr{L}_{n}^{I}\right)
$$

is one to one and onto.

Proof. The proof follows easily from ref. 21, II.2.7-II.2.9.

Let $R_{p}: S \mathscr{S}\left(\mathscr{L}_{n}^{I}\right) \rightarrow S \mathscr{S}\left(\mathscr{L}_{n-p}^{I}\right), p \in\{1, \ldots, n\}$ be a family of functions defined by

$$
\left(R_{p} F\right)\left(\Phi_{1}, \ldots, \Phi_{n-p}\right)=\mathrm{F}\left(\Phi_{1}, \ldots, \Phi_{n-p}, 0, \ldots, 0\right)
$$


where $\left(\Phi_{1}, \ldots, \Phi_{n}\right) \in \mathscr{L}_{n}^{I}, F \in S \mathscr{S}\left(\mathscr{L}_{n}^{I}\right)$ and we have used the convention $S \mathscr{S}\left(\mathscr{L}_{0}^{l}\right) \equiv \mathbf{C}$. It is easy to see that $R_{p} L_{n}^{I}=L_{n-p}^{I}$ for all $p \in\{1, \ldots, n\}$ and $n \in \mathbf{Z}^{+} \backslash\{0\}$ and $L_{p}^{I}\left(L_{n}^{I}\right)^{-1}=R_{n-p}$ for all $p \in\{1, \ldots, n\}$ and $n \in \mathbf{Z}^{+} \backslash\{0\}$ with $n \geqslant|I| / 2$.

\subsection{Supersymmetric Hilbert Spaces}

Let $\varphi: I \rightarrow \mathbf{R}^{2 n}$ be as in Definition 3.2 and let $K$ be a function from $I$ to $\left(\mathbf{Z}^{+}\right)^{2 n}$. We shall make use of the following notations:

$$
\begin{aligned}
\varphi^{K} & =\prod_{\substack{x \in I \\
p \in\{1, \ldots, n\}}}\left[\varphi_{p}^{1}(x)\right]^{K_{p}^{1}(x)}\left[\varphi_{p}^{2}(x)\right]^{K_{p}^{2}(x)} \\
\nabla_{K} & =\prod_{\substack{x \in I \\
p \in\{1, \ldots, n\}}} \frac{\partial^{K_{p}^{1}(x)}}{\partial\left[\varphi_{p}^{1}(x)\right]^{K_{p}^{1}(x)}} \frac{\partial^{K_{p}^{2}(x)}}{\partial\left[\varphi_{p}^{2}(x)\right]^{K_{p}^{2}(x)}} \\
|K| & =\sum_{\substack{x \in I \\
p \in\{1, \ldots, n\}}} K_{p}^{1}(x)+K_{p}^{2}(x)
\end{aligned}
$$

where $\quad \varphi_{p}(x)=\left(\varphi_{p}^{1}(x), \quad \varphi_{p}^{2}(x)\right), \quad x \in I, \quad p \in\{1, \ldots, n\}, \quad$ and $\quad K(x)=$ $\left(K_{1}(x), \ldots, K_{n}(x)\right)$, with $K_{p}(x)=\left(K_{p}^{1}(x), K_{p}^{2}(x)\right) \in\left(\mathbf{Z}^{+}\right)^{2} \forall p \in\{1, \ldots, n\}$.

Definition 3.13. Let $K_{1}, K_{2} \in\left(\mathbf{Z}^{+}\right)^{2 n|I|}$ and let $F \in S \mathscr{S}\left(\mathscr{L}_{n}^{I}\right)$. Let $n, I$ be as in Section 3.1, let $a \in S_{I}^{n}, \tau \in \mathbf{Z}^{+}$, and let

$$
\begin{aligned}
\|F\|_{a, n, I}^{K_{1}, K_{2}} & =\left\|2^{|a| / 2} \varphi^{K_{1}} \nabla_{K_{2}} F_{a}\right\|_{L^{2}\left(\mathbf{R}^{2 n i l \mid}\right)} \\
\left(\|F\|_{a, n, I}^{\tau}\right)^{2} & =\sum_{\left|K_{1}+K_{2}\right| \leqslant \tau}\left(\|F\|_{a, n, I}^{K_{1}, K_{2}}\right)^{2} \\
\left(\|F\|_{n, I}^{\tau}\right)^{2} & =\sum_{a \in S_{I}^{n}}\left(\|F\|_{a, n, I}^{\tau}\right)^{2}
\end{aligned}
$$

We define $\mathscr{H}_{\tau}\left(\mathscr{L}_{n}^{I}\right)$ to be the completion of $S \mathscr{S}\left(\mathscr{L}_{n}^{I}\right)$ under $\|\cdot\| \|_{n, I}^{t}$, where

$$
\left(\|F\|_{n, I}^{\tau}\right)_{\tau}^{2}=\sum_{p=0}^{n}\left(\left\|R_{p}(F)\right\|_{n-p, I}^{\tau}\right)^{2}
$$

and we have used the convention $\left\|R_{n} F\right\|_{0, I}^{\tau}=|F(0)|$ for all $\tau \in \mathbf{Z}^{+}$.

Trivially $R_{p}$ extends to a continuous linear function from $\mathscr{H}_{\tau}\left(\mathscr{L}_{n}^{I}\right)$ to $\mathscr{H}_{\tau}\left(\mathscr{L}_{n-p}^{I}\right)$ for all $\tau \in \mathbf{Z}^{+}$and for all $p \in\{1, \ldots, n\}$.

Definition 3.14. Let $\tau \in \mathrm{Z}^{+}$and let $S M_{I}^{+}=L_{1}^{I}\left[\mathscr{S}\left(M_{I}^{+}\right)\right]$, where

$$
\mathscr{P}\left(M_{I}^{+}\right)=\bigcap_{n \in \mathrm{N}} \mathscr{S}^{n}\left(M_{I}^{+}\right)
$$


We define $\mathscr{H}_{\tau}^{I}$ to be the completion of $S M_{I}^{+}$under the norm $\|\cdot\|_{I}^{\tau}=$ $\|\cdot\| \|_{1, I}^{i}$.

It is clear that $\mathscr{H}_{\tau}^{I}$ is a subspace of $\mathscr{H}_{\tau}\left(\mathscr{L}_{n}^{I}\right)$ and it consists of vectorvalued functions $F=\left\{F_{a}\right\}_{a \in S_{I}^{1}}$ with $F_{a} \in L^{2}\left(\mathbf{R}^{2|| l \mid}\right)$ for all $a \in S_{I}^{1}$. However, in view of the next proposition, we can assume that the components of $F$ are in some sense weak derivatives of $F_{a_{\varnothing \varnothing}}$.

Proposition 3.15. Let $\tau \in \mathbf{Z}^{+}$and let $F, G \in \mathscr{H}_{\tau}^{I}$; then $F_{a}=G_{a}$ for all $a \in S_{I}^{n} \Leftrightarrow F_{a_{\varnothing}}=G_{a_{\varnothing \varnothing}}$.

Proof. Since $\mathscr{H}_{\tau^{\prime}}^{I}$ is a subspace of $\mathscr{H}_{\tau}^{I}$ for $\tau \leqslant \tau^{\prime}$, it suffices to prove the result for $\mathscr{H}_{0}^{\tau}$.

Let $F$ be an element of $\mathscr{H}_{\tau}^{I}$ with $F_{a \varnothing}=0$ and let $\left\{f^{k}\right\}_{k \in \mathbf{N}}$ be a sequence in $\mathscr{S}\left(M_{I}^{+}\right)$with $L_{1}^{I}\left(f^{k}\right) \rightarrow F$ in $\mathscr{H}_{\tau}^{T}$ as $k \rightarrow+\infty$. It follows from the usual chain rule that for all $x \in I, \varphi \in \mathbf{R}^{2|I|}$, and $k \in \mathbf{N}$

$$
\nabla_{x}\left[L_{1}^{I}(f)^{k}\right](\varphi)=2 \varphi(x)\left[\frac{\partial f^{k}}{\partial A_{x x}}\right]\left(A_{\varphi}\right)+\sum_{x<y} \varphi(y)\left[\frac{\partial f^{k}}{\partial A_{x y}}\right]\left(A_{\varphi}\right)
$$

where $\nabla_{x}$ is the usual gradient with respect to the variable $\varphi(x) \in \mathbf{R}^{2}$.

Trivially for every $k \in \mathbf{N}$ we can solve the system of equations above and compute the partial derivatives

$$
\partial f^{k} / \partial A_{x y}, \quad x \leqslant y
$$

in terms of $\nabla_{x}\left[L_{1}^{I}\left(f^{k}\right)\right], x \in I$, on all of $\mathbf{R}^{2|I|}$ except maybe a hypersurface which we will denote by $H_{k}$.

Let $H=\bigcup_{k \in \mathbf{N}} H_{k}$ and let $j$ be a function from $\mathscr{S}\left(M_{I}^{+}\right)$whose support is a compact subset of the interior of $M_{I}^{+} \backslash\left\{M \in M_{I}^{+}: M=A_{\varphi}\right.$ for some $\varphi \in H\}$ and let $J$ be the complex-valued function on $\mathbf{R}^{2|I|}$ which is defined by

$$
J(\varphi)=j(A \varphi), \quad \varphi \in \mathbf{R}^{2|\eta|}
$$

Using successive integrations by parts, one can show that, $\forall a \in S_{I}^{1}$,

$$
\begin{aligned}
\left(F_{a}, J\right) & =\lim _{k \rightarrow \infty}\left(\left[L_{1}^{I} f^{k}\right]_{a}, J\right) \\
& =\lim _{k \rightarrow \infty} \int_{\mathbf{R}^{2|| \mid}} \partial_{a} f\left(A_{\varphi}\right) J(\varphi) d^{2|I|} \varphi \\
& =\lim _{k \rightarrow \infty} \int_{\mathbf{R}^{2|| \mid}} f^{k}\left(A_{\varphi}\right) J^{\prime}(\varphi) d^{2|I|} \varphi
\end{aligned}
$$


where $J^{\prime}$ is some other function in $\mathscr{S}\left(\mathbf{R}^{2|I|}\right)$ whose support is a compact subset of the interior of $\mathbf{R}^{2|x|} \backslash H$.

Since $\left[L_{1}^{I} f^{K}\right]_{a_{\varnothing}} \rightarrow 0$ in $L^{2}\left(\mathbf{R}^{2|I|}\right)$ as $k \rightarrow \infty$ and $j$ was chosen arbitrarily, we conclude that $F=0$ and the result follows.

\section{THE SUPERSYMMETRIC TRANSFER MATRIX}

\subsection{The Operators $T, B(z)$}

Let $m \in \mathbf{Z}^{+} \backslash\{0\}$ and let $M_{m}^{+}, S_{m}, \mathscr{L}^{m}, S M_{m}^{+}$, and $\mathscr{H}_{\tau}^{m}$ denote the sets $M_{\{1, \ldots, m\}}^{+}, S_{\{1, \ldots, m\}}^{1}, \mathscr{L}_{1}^{\{1, \ldots, m\}}, S M_{\{1, \ldots, m\}}^{+}$, and $\mathscr{H}_{t}^{\{1, \ldots, m\}}$, respectively.

Definition 4.1, We define the supersymmetric transfer matrix as an operator $T$ from $S M_{m}^{+}$to $S \mathscr{S}\left(\mathscr{L}^{m}\right)$ defined by ${ }^{(21)}$

$$
[T(F)]\left(\Phi^{\prime}\right)=\int \exp \left[i \sum_{j=1}^{m} \Phi(j) \cdot \Phi^{\prime}(j)\right] F(\Phi) d \Phi, \quad \Phi^{\prime} \in \mathscr{L}^{M}
$$

Note that in view of Definition 3.3 the integral in (4.1) is well defined, since $\exp \left[i \sum_{j=1}^{m} \Phi(j) \cdot \Phi^{\prime}(j)\right]$ is a kernel which is naturally defined in $\mathscr{L}^{m} \times \mathscr{L}^{m}$.

Definition 4.2. Let $\tau \in \mathbf{Z}^{+}$and let $h$ be a complex-valued function defined on $\mathbf{R}$; we will say that $h \in \mathscr{G}^{\tau}$ if and only if $h$ is bounded, of class $C^{\tau}$ on $(0,+\infty)$ with bounded derivatives, and $\left|h^{\left(\tau^{\prime}\right)}(r)\right| \rightarrow 0$ as $r \rightarrow \infty$ for all $\tau^{\prime}$ with $\tau^{\prime} \leqslant \tau$.

Let $z \in \bar{\Pi}^{+}=\{z \in \mathbf{C}: \operatorname{Im} z \geqslant 0\}$ and let

$$
V_{Z}^{t}=\left(\begin{array}{ccc}
v(t, 1)-z & 0 \\
& \ddots & \\
0 & v(t, m)-z
\end{array}\right)+\left(\begin{array}{ccccc}
0 & -1 / 2 & & & 0 \\
-1 / 2 & 0 & -1 / 2 & & \\
& \ddots & \ddots & \ddots & \\
& & & 0 & -1 / 2 \\
0 & & & -1 / 2 & 0
\end{array}\right)
$$

where $\mathscr{D}_{m} \ni(t, j) \rightarrow V(t, j) \in \mathbf{R}$ is the random potential defined in Section 2 . Let $b_{t}(z): M_{m}^{+} \rightarrow \mathbf{C}$ be a complex-valued function defined by

$$
b_{t}(z)(A)=\exp \left[-\operatorname{itr}\left(V_{z}^{t} A\right)\right], \quad A \in M_{m}^{+}, \quad t \in \mathbf{Z}
$$

Let $b(z): M_{m}^{+} \rightarrow \mathbf{C}$ be a complex-valued function defined by

$$
b(z)(A)=\prod_{k=1}^{m} h\left(A_{k k}\right) \prod_{k=1}^{m-1} \exp \left(i A_{k, k+1}\right), \quad A \in M_{m}^{+}
$$


Let $\beta_{t}(z): \mathscr{L}^{m} \rightarrow \Lambda\left(\mathbf{R}^{2 m}\right)$ be a superfunction defined by

$$
\beta_{t}(z)(\Phi)=b_{t}(z)\left(A_{\Phi}\right), \quad \Phi \in \mathscr{L}^{m}, \quad t \in \mathbf{Z}
$$

Let us assume that $h$ is of class $C^{m}$ with bounded derivatives and let $\beta(z)$ : $\mathscr{L}^{m} \rightarrow A\left(\mathbf{R}^{2 m}\right)$ be the superfunction defined by

$$
\beta(z)(\Phi)=b(z)\left(A_{\Phi}\right), \quad \Phi \in \mathscr{L}^{m}
$$

One can easily check that $\beta(z) \in S C\left(\mathscr{L}^{m}\right)$ and that if $\int|v|^{m} \mu(d v)<+\infty$, then

$$
\mathbf{E}\left\{\beta_{t}(z)\right\}=\beta(z), \quad t \in \mathbf{Z}
$$

We will denote by $B(z)$ and $B_{t}(z)$ the operators multiplication by $\beta(z)$ and $\beta_{t}(z)$, respectively. then:

Proposition 4.3. Let $m \in \mathbf{Z}^{+} \backslash\{0\}$ and let $h \in \mathscr{G}^{\tau}$ for some $\tau \in \mathbf{Z}^{+}$;

(i) $B(z)$ is a bounded operator on $\mathscr{H}_{\tau}^{m}$, for all $z \in \bar{\Pi}^{+}$.

(ii) $T$ extends to a bounded operator on $\mathscr{H}_{\tau}^{m}$. In particular, $T$ is an isometry in $\mathscr{H}_{0}^{m}$ and $T^{2}=I$.

(iii) $B(z) T B(z)$ is a bounded operator from $\mathscr{H}_{\tau^{\prime}}^{m}$ to $\mathscr{H}_{\tau^{\prime}+1}^{m}$ for all $\tau^{\prime}<\tau$ and $z \in I I^{+}=\{z \in \mathbf{C}: \operatorname{Im} z>0\}$.

Proof. In view of ref. 21 , IV.1.4, it suffices to show that $\mathscr{H}_{\tau^{\prime}}^{m}$ is left invariant by $T$ and $B(z), z \in \bar{\Pi}^{+}$for all $\tau^{\prime} \leqslant \tau$. Indeed, let $n \in \mathbf{Z}^{+}$with $n \geqslant m / 2$, let $L_{n}^{m}$ denote the map $L_{n}^{\{1, \ldots, m\}}$, and let $\mathscr{L}_{n}^{m}$ denote the space $\mathscr{L}_{n}^{\{1, \ldots, m\}}$. For a fixed $f \in S M_{m}^{+}$we set

$$
F^{n}\left(\Phi^{\prime}\right)=\int \exp \left[i \sum_{j=l}^{m} \Phi(j) \cdot \Phi^{\prime}(j)\right]\left(L_{n}^{m} f\right)(\Phi) d \Phi, \quad \Phi^{\prime} \in \mathscr{L}_{n}^{m}
$$

where the integration above is over the superspace $\mathscr{L}_{n}^{m}$.

It is a direct consequence of ref. 21 , II.2.10, that

$$
\left[R_{n-1} F^{n}\right](\Phi)=T\left[L_{1}^{m} f\right](\Phi), \quad \Phi \in \mathscr{L}^{m}
$$

Moreover, one can easily check that $F^{n} \in S \mathscr{S}\left(\mathscr{L}_{n}^{n}\right)$ (see ref. 21, III.2.1) and that for all $\Phi \in \mathscr{L}^{m}$

$$
L_{1}^{m}\left[\left(L_{n}^{m}\right)^{-1}\left(F^{n}\right)\right](\Phi)=T\left[L_{1}^{m} f\right](\Phi)
$$

Thus, $T\left(L_{1}^{m} f\right) \in \mathscr{S}^{n}\left(M_{m}^{+}\right)$. Since $n$ was chosen arbitrarily large, we conclude that $T\left(L_{1}^{m} f\right) \in \mathscr{S}\left(M_{m}^{+}\right)$and the result for $T$ follows. 
Finally, the fact that $B(z), z \in \bar{\Pi}^{+}$, leaves $\mathscr{H}_{\tau^{\prime}}^{m}, \tau^{\prime} \leqslant \tau$, invariant follows through an easy approximation argument.

Proposition 4.4. Let $h \in \mathscr{G}^{\tau}$ for some $\tau \in \mathbf{Z}^{+} \backslash\{0\}$ and let $m \in \mathbf{Z}^{+} \backslash\{0\}$; then:

(i) $(B(z) T)^{2}$ is compact on $\mathscr{H}_{\tau}^{m}$ for all $z \in \bar{\Pi}^{+}$.

(ii) Let $B\left(\mathscr{H}_{\tau}^{m}\right)$ be the Banach space of all bounded linear operator on $\mathscr{H}_{\tau}^{m}$. Then the operator-valued function $\bar{\Pi}^{+} z \rightarrow B(z) T B(z) \in B\left(\mathscr{H}_{\tau}^{m}\right)$ is norm continuous.

Proof. It follows directly from the previous proposition and ref. 21, IV.1.6.

\subsection{The Spectrum of $B(z) T$}

We first state a theorem the proof of which follows directly from Propositions 4.3 and 4.4 .

Theorem 4.5. Let $m \in \mathbf{Z}^{+} \backslash\{0\}$ and let $h \in \mathscr{G}^{t}$ for some $\tau \in \mathbf{Z}^{+}$; then:

(i) $B(z) T$ has discrete spectrum in $\mathscr{H}_{\tau^{\prime}}^{m}$ with zero being the only possible accumulation point for all $z \in \bar{\Pi}^{+}$and all $\tau^{\prime} \leqslant \tau$.

(ii) Let $z \in \Pi^{+}$and let $\tau^{\prime} \leqslant \tau$. A superfunction $F$ is an eigenvector of $B(z) T$ on $\mathscr{H}_{0}^{m} \Leftrightarrow F$ is an eigenvector of $B(z) T$ on $\mathscr{H}_{t^{\prime}}^{m}$.

We now proceed to study in more detail the point spectrum of $B(z) T$ on the Hilbert space $\mathscr{H}_{\tau}^{m}$. In particular, if $h \in \mathscr{G}^{\tau}$, we will study the existence and uniqueness of the eigenvalue 1 for the operators $B(z) T$, $z \in \bar{\Pi}^{+}$.

Theorem 4.6. Let $m \in \mathbf{Z}^{+} \backslash\{0\}$ and let $h \in \mathscr{G}^{\tau}$ for some $\tau \in \mathbf{Z}^{+}$; then:

(i) 1 is an eigenvalue of $B(z) T$ on $\mathscr{H}_{\tau^{\prime}}^{m}$ for all $z \in \bar{\Pi}^{+}$and all $\tau^{\prime} \leqslant \tau$.

(ii) If $z \in \Pi^{+}$, then 1 is algebraically simple and it is the only eigenvalue of modulus 1 .

(iii) The spectral radius of $B(z) T$ on $\mathscr{H}_{\tau^{\prime}}^{m}$ is 1 for all $z \in \bar{\Pi}^{+}$and all $\tau^{\prime} \leqslant \tau$.

Proof. Let $z \in \bar{\Pi}^{+}$and let $F \in S M_{m}^{+}$. As in ref. 21, Theorem IV.2.12, one can show that

$$
\lim _{k \rightarrow \infty}(B(z) T)^{k}(F)
$$


exists in $\mathscr{H}_{0}^{m}$ and it is equal to $F(0) \Xi(z)$, where $\Xi(z)$ is a fixed vector in $\mathscr{H}_{0}^{m}$ which is independent of $F$ and has the property $[\Xi(z)](0)=1$.

Thus, (i)-(iii) in the case $z \in \Pi^{+}$follow from Theorem 4.5. The rest of the theorem now follows easily from Proposition 4.4(ii) and Theorem 4.5(i).

Let $H$ be the Hamiltonian defined in Section 2 and let $E \in \mathbf{R}$. To the stationary Schrödinger equation

$$
(H-E) \psi=0
$$

we associate the matrix equation ${ }^{(23,24)}$

$$
-\frac{1}{2} M_{n-1}-\frac{1}{2} M_{n+1}+V_{E}^{n} M_{n}=0
$$

where $M_{n}, n \in \mathbf{N}$, are $m \times m$ square matrices.

Let $H_{[1,+\infty)}$ be the Hamiltonian $H$ with boundary conditions $u(x, y)=0$ if $(x, y) \in \mathscr{D}_{m}$ with $x \notin[1,+\infty)$. If $\psi$ is a solution of the equation

$$
\left(H_{[1,+\infty)}-E\right) \psi=0
$$

then it can be written as

$$
\psi_{n}=P_{n} \psi_{1}, \quad n=1,2, \ldots
$$

where $P_{n}, n=1,2, \ldots$, is the solution of the associated matrix equations (4.4) which is given by

$$
\left(\begin{array}{c}
P_{n+1} \\
P_{n}
\end{array}\right)=\left(\begin{array}{cc}
2 V_{E}^{n} & -I \\
I & 0
\end{array}\right) \cdots\left(\begin{array}{cc}
2 V_{E}^{1} & -I \\
I & 0
\end{array}\right)\left(\begin{array}{l}
I \\
0
\end{array}\right)
$$

where $I$ is the $m \times m$ identity matrix, 0 the $m \times m$ zero matrix, and

$$
\psi_{n}=\left(\begin{array}{c}
\psi(m, n) \\
\vdots \\
\psi(1, n)
\end{array}\right), \quad n \in \mathbf{N}
$$

Let $n \in \mathbf{Z}^{+} \backslash\{0,1\}$. We will denote by $\Gamma_{n}$ the operator on $l^{2}\left(\mathscr{D}_{m}\right)$ which is defined by

where

$$
\left(\Gamma_{n} u\right)(x)=\sum_{y \in \mathscr{I}_{m}} \Gamma_{n}(x, y) u(y), \quad u \in l^{2}\left(\mathscr{D}_{m}\right)
$$

$$
\Gamma_{n}(x, y)= \begin{cases}1 & \text { if } x=y=(i, n) \text { for some } i=1,2,3, \ldots \\ 0 & \text { otherwise }\end{cases}
$$


Let $\varepsilon>0$. We shall make use of the operator $H_{[1, n]}^{\varepsilon}$, which is defined by

$$
H_{[1, n]}^{\varepsilon}=H_{[1, n]}-i \varepsilon \Gamma_{n}
$$

Lemma 4.7. The resolvent of $H_{[1, n]}^{\varepsilon}$ contains the set of real numbers for all configurations of the potential, all $n \in \mathbf{Z}^{+} \backslash\{0,1\}$, and $\varepsilon>0$.

Proof. Let $E \in \mathbf{R}, \varepsilon>0, n \in \mathbf{Z}^{+} \backslash\{0,1\}$, and let $\psi$ be a solution of $\left(H_{[1, n]}^{e}-E\right) \psi=0$ with $\psi(k, 1) \in \mathbf{R}$ for all $k=1,2, \ldots, m$. It is an immediate consequence of (4.5) that $\psi$ is real-valued. However, since

with

$$
V_{E}^{n} \psi_{n}-\frac{1}{2} \psi_{n-1}-i \varepsilon \psi_{n}=0
$$

$$
\psi_{j}=\left(\begin{array}{c}
\psi_{(m, j)} \\
\vdots \\
\psi_{(1, j)}
\end{array}\right), \quad j=1,2, \ldots, n
$$

we conclude that $\psi_{n}=\psi_{n-1}=0$. Thus, $\psi \equiv 0$ and the result follows.

Let $E \in \mathbf{R}$, we will denote by $G_{[1, n]}^{\varepsilon}(E)$ the operator $\left(H_{[1, n]}^{\varepsilon}-E\right)^{-1}$. We will also denote by $G_{[1, n]}^{\varepsilon}\left(E, k, k^{\prime}\right)$ the matrix

$$
G_{[1, n]}^{\varepsilon}\left(E, k, k^{\prime}\right)=\left(\left\langle(k, i)\left|G_{[1, n]}^{\varepsilon}(E)\right|\left(k^{\prime}, j\right)\right\rangle\right)_{i, j=1,2, \ldots, m}
$$

where $k, k^{\prime} \in\{1,2, \ldots, n\}$.

Lemma 4.8. Let $E \in \mathbf{R}$. For all configurations of the potential the following are true:

(i) $\operatorname{Im} G_{[1, n]}^{\varepsilon}(E)$ is positive definite for all $\varepsilon>0$.

(ii) $P_{n+1}-i \varepsilon P_{n}$ is an invertible $m \times m$ matrix for all $n \in \mathbf{Z}^{+} \backslash\{0,1\}$ and $0<\varepsilon$, and

$$
G_{[1, n]}^{\varepsilon}(E, k, n)=2 P_{k}\left(P_{n+1}-i 2 \varepsilon P_{n}\right)^{-1}
$$

for all $n, k \in \mathbf{Z}^{+} \backslash\{1,0\}$ with $k \leqslant n$ and $\varepsilon>0$.

Proof. (i) This follows easily from the resolvent identity.

(ii) Since

$$
\left(\begin{array}{cc}
2 V_{E}^{j} & -I \\
I & 0
\end{array}\right), \quad j=1,2, \ldots
$$

are symplectic matrices, we conclude that $P_{j+1} P_{j}^{\prime}=P_{j} P_{j+1}^{\prime}$ for all $j=1$, $2, \ldots$, where $P_{j}^{\prime}$ denotes the transpose of $P_{j}$. Hence

$$
\left(P_{j+1}-i \varepsilon P_{j}\right)\left(P_{j+1}^{\prime}+i \varepsilon P_{j}^{\prime}\right)=P_{j+1} P_{j+1}^{\prime}+\varepsilon^{2} P_{j} P_{j}^{\prime}, \quad j=1,2, \ldots
$$


One can easily check that since

$$
\operatorname{det}\left(\begin{array}{cc}
2 V_{E}^{j} & -I \\
I & 0
\end{array}\right) \neq 0
$$

for all $j=1,2, \ldots$, the determinant of $P_{j+1} P_{j+1}^{\prime}+P_{j} P_{j}^{\prime}$ is also nonzero for all $j=1,2, \ldots$. Furthermore,

$$
\left|\operatorname{det}\left(P_{j+1}-i \varepsilon P_{j}\right)\right|^{2} \geqslant \min \left(1, \varepsilon^{2}\right) \operatorname{det}\left[P_{j+1} P_{j+1}^{\prime}+P_{j} P_{j}^{\prime}\right]
$$

for all $j=1,2, \ldots$ and all $\varepsilon>0$. Thus, the invertibility of $P_{j+1}-i \varepsilon P_{j}$ follows.

Finally, let $\psi$ be a solution of $\left(H_{[1,+\infty)}-E\right) \psi=0$ and let

$$
\psi_{j}=\left(\begin{array}{c}
\psi_{(m, j)} \\
\vdots \\
\psi_{(1, j)}
\end{array}\right), \quad j=1,2, \ldots
$$

One can easily check that (also see ref. 28)

$$
\psi_{k}=\left[G_{[1, n]}^{\varepsilon}(E, k, n)\right]\left(\frac{1}{2} \psi_{n+1}-i \varepsilon \psi_{n}\right), \quad k=1,2, \ldots
$$

Thus,

$$
P_{k}=G_{[1, n]}^{\varepsilon}(E, k, n)\left(\frac{1}{2} P_{n+1}-i \varepsilon P_{n}\right)
$$

and the result follows.

Proposition 4.9. Let $E \in \mathbf{R}$ and let $\varepsilon>0$.

(i) Let $h(r) \rightarrow 0$ as $r \rightarrow \infty$; then $\left\|G_{[1, n]}^{\varepsilon}(E, 1, n)\right\| \rightarrow 0$ almost surely as $n \rightarrow \infty$.

(ii) $\left\|G_{[1, n]}^{\varepsilon}(E, n, n)\right\| \leqslant M$ for all $n \in \mathbf{Z}^{+} \backslash\{1,0\}$ and for some $M<+\infty$ independent of $n$.

Proof. (i) Under the assumption that $h$ decays at infinity, it follows from ref. $23, \mathrm{I} .3$ and 1.2 , that $\operatorname{tr}\left[\left(P_{n+1} P_{n+1}^{\prime}+P_{n} P_{n}^{\prime}\right)^{-1}\right] \rightarrow 0$ almost surely as $n \rightarrow \infty$. ${ }^{(20,23)}$

One can easily show, however, that for all $0<\varepsilon<1$,

$$
\operatorname{tr}\left[\left(P_{n+1} P_{n+1}^{\prime}+\varepsilon^{2} P_{n} P_{n}^{\prime}\right)^{-1}\right] \leqslant \frac{1}{\varepsilon^{2}} \operatorname{tr}\left[\left(P_{n+1} P_{n+1}^{\prime}+P_{n} P_{n}^{\prime}\right)^{-1}\right]
$$

and (i) follows from (4.6).

(ii) This follows from (4.6) and the inequality

$$
\operatorname{tr}\left[P_{n} P_{n}^{\prime}\left(P_{n+1} P_{n+1}^{\prime}+\varepsilon^{2} P_{n} P_{n}^{\prime}\right)^{-1}\right] \leqslant \frac{1}{\varepsilon^{2}} m
$$


Let $A$ be an $m \times m$ matrix and let $\Phi, \Phi^{\prime} \in \mathscr{L}^{m}$. We will be using the notations

$$
(A \Phi)(x)=\sum_{y \in\{1, \ldots, m\}} A_{x y} \Phi(y), \quad x \in\{1, \ldots, m\}
$$

and

$$
\left\langle\Phi, \Phi^{\prime}\right\rangle=\sum_{x \in\{1, \ldots, m\}} \Phi(x) \cdot \Phi^{\prime}(x)
$$

We will also be using the notations

$$
(A \varphi)(x)=\sum_{y \in\{1, \ldots, m\}} A_{x y} \varphi(y), \quad x \in\{1, \ldots, m\}
$$

and

$$
\left\langle\varphi, \varphi^{\prime}\right\rangle=\sum_{x \in\{1, \ldots, m\}} \varphi(x) \cdot \varphi^{\prime}(x)
$$

where $\Phi(x)=(\varphi(x), \bar{\psi}(x), \psi(x)), \Phi^{\prime}(x)=\left(\varphi^{\prime}(x), \bar{\psi}^{\prime}(x), \psi^{\prime}(x)\right)$.

Lemma 4.10. Let $m \in \mathbf{Z}^{+} \backslash\{0\}$, let $E \in \mathbf{R}, \quad \eta \in \mathbf{R}^{+} \backslash\{0\}$, let $f \in \mathscr{S}\left(M_{m}^{+}\right)$with $f(0)=0$, and let $F=L_{1}^{m} f$; then

$$
\left(\left[B(E+i \eta) T(B(E) T)^{k} B(E+i \eta) T\right](F)\right)_{a_{\varnothing}} \rightarrow 0
$$

in $L^{2}\left(\mathbf{R}^{2 m}\right)$ as $k \rightarrow \infty$.

Proof. Through an easy approximation argument and III.2.3 of ref. 21, we conclude that for all $\Phi \in \mathscr{L}^{m}$ and almost all configurations of the potential

$$
\begin{aligned}
{\left[B_{0}(E+\right.} & \left.i \eta) T B_{1}(E) T \cdots B_{k}(E) T B_{k+1}(E+i \eta) T\right](F)(\Phi) \\
= & \exp \left[-i\left\langle\Phi, V_{E+i \eta}^{1} \Phi\right\rangle+\frac{i}{4}\left\langle\Phi, G_{[1, k+1]}^{\eta}(E ; 1,1) \Phi\right\rangle\right] \\
& \times \int \exp \left[\frac{i}{4}\left\langle\Phi, G_{[1, k+1]}^{\eta}(E ; 1, k+1) \Phi^{\prime}\right\rangle\right. \\
& +\frac{i}{4}\left\langle\Phi^{\prime}, G_{[1, k+1]}^{\eta}(E ; k+1,1) \Phi\right\rangle \\
& \left.+\frac{i}{4}\left\langle\Phi^{\prime}, G_{[1, k+1]}^{\eta}(E ; k+1, k+1) \Phi^{\prime}\right\rangle\right] F\left(\Phi^{\prime}\right) d \Phi^{\prime}
\end{aligned}
$$


Using ref. 21 , II.2.10, one can rewrite (4.7) as

$$
\begin{aligned}
\left(\left[B_{0}(E\right.\right. & \left.\left.+i \eta) T B_{1}(E) T \cdots B_{k}(E) T B_{k+1}(E+i \eta) T\right](F)\right)_{a_{\varnothing}}(\varphi) \\
= & \exp \left[-i\left\langle\varphi, V_{E+i \eta}^{1} \varphi\right\rangle+\frac{1}{4} i\left\langle\varphi, G_{[1, k+1]}^{\eta}(E, 1,1) \varphi\right\rangle\right] \\
& \times \int_{\mathbf{R}^{2 m}}\left[\operatorname { e x p } \frac { i } { 4 } \left\{\left\langle\varphi, G_{[1, k+1]}^{\eta}(E, 1, k+1) \varphi^{\prime}\right\rangle\right.\right. \\
& \left.\left.+\left\langle\varphi^{\prime}, G_{[1, k+1]}^{\eta}(E ; k+1,1) \varphi\right\rangle\right\}-1\right] \\
& \times \partial_{a_{m}}\left(J_{k}^{\eta} f\right)\left(A_{\varphi^{\prime}}\right) d^{2 m} \varphi^{\prime}
\end{aligned}
$$

where $a_{m}=a_{\{1, \ldots, m\}} \in S^{m}$ and $J_{k}^{n}$ an element of $C^{\infty}\left(M_{m}^{+}\right)$which is defined by

$$
J_{k}^{n}(D)=\exp \left[-\frac{i}{4} \operatorname{tr}\left[D G_{[1, k+1]}^{\eta}(E, k+1, k+1)\right]\right], \quad D \in M_{m}^{+}
$$

It now follows from (4.8) and ref. 21, III.2.5, that for almost all configurations of the potential

$$
\begin{aligned}
& \|\left(\left[B_{0}(E+i \eta) T B_{1}(E) T\right.\right. \\
& \left.\left.\quad \cdots B_{k}(E) \mathbf{T} B_{k+1}(E+i \eta) T\right](F)\right)_{a_{\varnothing}} \|_{L^{2}\left(\mathbf{R}^{2 m}\right)} \rightarrow 0
\end{aligned}
$$

as $k \rightarrow \infty$. On the other hand, from (4.8) and Proposition 4.9 one can easily conclude that

$$
\begin{aligned}
& \|\left(\left[B_{0}(E+i \eta) T B_{1}(E) T\right.\right. \\
& \left.\left.\quad \cdots B_{k}(E) T B_{k+1}(E+i \eta) T\right](F)\right)_{a \not \varnothing} \|_{L^{2}\left(\mathbf{R}^{2 m}\right)} \leqslant M
\end{aligned}
$$

for some $M<+\infty$ which is independent of $k$ and of the configuration of the potential. Thus, the result follows from the bounded convergence theorem and the independence of $V_{z}^{i}, i \in \mathbf{Z}, z \in \bar{\Pi}^{+}$.

Theorem 4.11. Let $E \in \mathbf{R}, m \in \mathbf{Z}^{+} \backslash\{0\}, \tau \in \mathbf{Z}^{+}$, and let $h \in \mathscr{G}^{\tau}$; then 1 is an algebraically simple eigenvalue of $B(E) T$ on $\mathscr{H}_{\tau^{\prime}}^{m}$ for all $0 \leqslant \tau^{\prime} \leqslant \tau$.

Proof. In view of the fact that $\mathscr{H}_{\tau^{\prime}}^{m}$ is a subspace of $\mathscr{H}_{\tau}^{m}$ whenever $\tau^{\prime} \leqslant \tau$, we have only to show that 1 is algebraically simple on $\mathscr{H}_{0}^{m}$.

Indeed, let $\lambda_{1}, \ldots, \lambda_{n}$ be the eigenvalues of $(B(E) T)^{2}$ on $\mathscr{H}_{0}^{m}$ which lie on the unit circle. Let $P_{1}, \ldots, P_{n}$ be the corresponding spectral projections and let $D_{1}, \ldots, D_{n}$ be the corresponding nilpotents that appear in the Jordan 
decomposition of $(B(E) T)^{2}{ }^{(30)}$ Since $(B(E) T)^{2} P_{i}=\lambda_{i} P_{i}+D_{i}$, one can easily see that for all $k \in \mathbf{Z}^{+}$,

$$
(B(E) T)^{2 k}=Q^{k}+\sum_{j=1}^{n}\left[\lambda_{j}^{k} P_{j}+\sum_{m=1}^{m_{j}} \lambda_{j}^{k-m} D_{j}^{m}\left(\begin{array}{c}
k \\
m
\end{array}\right)\right]
$$

where $Q$ is an operator on $\mathscr{H}_{0}^{m}$ whose spectral radius is strictly less than one and $m_{j}$ is the algebraic multiplicity of $\lambda_{j}$.

Let $F \in S M_{m}^{+}$with $F(0)=0$. If $u>0$, then Lemma 4.10 implies that

$$
\|\left(\left[B(E+i \eta) T(B(E) T)^{2 k+2} B(E+i \eta) T\right](\mathbf{F})_{a \not b} \|_{L^{2}\left(\mathbf{R}^{2 m}\right)} \rightarrow 0\right.
$$

as $k \rightarrow \infty$ and since

$$
\lim _{k \rightarrow \infty}\left\|Q^{k}(F)\right\|_{\{1, \ldots, m\}}^{0}=0
$$

we conclude that

$$
\left[B(E+i \eta) T B(E) T P_{j}(E) B(E) T B(E+i \eta) T\right](F)=0
$$

for all $\eta>0$ and all $j=1,2, \ldots, n$.

Let $A_{0}^{m}$ be the closure of $\left\{F \in S M_{m}^{+}: F(0)=0\right\}$ in $\mathscr{H}_{0}^{m}$. It is easy to see that $A_{0}^{m}$ is invariant under the action of $B(E) T$ and has codimension one. Letting $\eta \rightarrow 0$, it follows from (4.9) that $P_{j}(F)=0$ for all $F \in A_{0}^{m}$ and all $j=$ $1,2, \ldots, n$. Thus, 1 has geometric multiplicity one.

The rest of the proof now follows as in ref. 21, IV.2.12(iii).

\section{CONCLUSION}

In this section we give a brief outline of the rest of the proof of Theorems 2.1 and 2.2. As we have already pointed out, we use the same techniques as in ref. 21 and we refer the reader to the last sections of that article for more details.

Let $E_{0} \in \mathbf{R}, \tau \in \mathbf{Z}^{+} \backslash\{0\}$, and $h \in \mathscr{G}^{\tau}$. Let $z \in \mathbf{C}$, let $m$ be the width of the strip $\mathscr{D}_{m}$, and let $\Xi(z)$ be the eigenvector of $B(z) T$ on $\mathscr{H}_{t}^{m}$ which corresponds to 1 and has the property $\Xi(z)(0)=1$. From Theorems 4.5, 4.6 , and 4.11 and a routine perturbation argument, ${ }^{(12-14,21)}$ we conclude that there exists a closed contour $\gamma$ around 1 and a complex neighborhood $U_{E_{0}}$ of $E_{0}$ such that $\gamma$ is in the resolvent of $B(z) T$ for all $z \in U_{E_{0}} \cap \bar{\Pi}^{+}$and

$$
\Xi(z)=\frac{1}{2 \pi i}\left[\int_{\tau}\left(B(z) T-z^{\prime}\right)^{-1} d z^{\prime}\right]\left(\Xi\left(E_{0}\right)\right)
$$

One can easily show now (see ref. 21, V.1.2) that the complex-valued function

$$
\bar{\Pi}^{+} \ni z \rightarrow \Xi(z) \in \mathscr{H}_{0}^{m}
$$




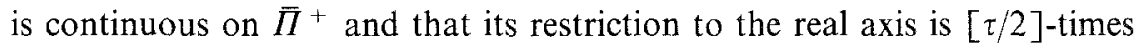
norm continuously differentiable. Thus, Theorem 2.1 follows directly from the relations ${ }^{(21)}$

$$
\begin{aligned}
\frac{d N_{a c}}{d E}= & \frac{1}{\pi} \lim _{n \searrow 0} \operatorname{Im} \sum_{j=1}^{m} \mathbf{E}\left\{\left\langle(0, j)\left|(H-E+i)^{-1}\right|(0, j)\right\rangle\right\} \\
= & \frac{1}{\pi} \lim _{n \searrow 0} \operatorname{Im} \sum_{j=1}^{m} i \int \bar{\psi}(0, j) \psi(0, j) \\
& \times[\Xi(E+i \eta)](\Phi)[T(\Xi(E+i \eta))](\Phi) d \Phi
\end{aligned}
$$

where $N_{a c}$ is the absolutely continuous part of $N$ and the integration is over the superspace $\mathscr{L}^{m}$.

Finally, under the assumptions of Theorem 2.2 one can easily show that the complex-valued function

$$
\bar{\Pi}^{+} \ni z \mapsto \Xi(z) \in \mathscr{H}_{0}^{m}
$$

has an analytic extension to $\{z \in \mathbf{C}: \operatorname{Im} z>-b\}$ for some $b>0$ (see ref. 21 , V.1.4). Thus, Theorem 2.2 follows again from relations (5.2).

\section{ACKNOWLEDGMENT}

The work of A.K. was partially supported by the NSF under grant DMS 87-02301.

\section{REFERENCES}

1. P. Anderson, Phys. Rev. 109:1492 (1958).

2. R. Carmona, Random Schrödinger Operators, Proceedings of the Ecole d'été de Probabilité XIV Saint-Flour 1984 (Lecture Notes in Mathematics, Vol. 1180, Springer, Berlin, 1986).

3. W. Kirsch and F. Martinelli, On the density of states of Schrödinger operators with a random potential, J. Phys. A 15:2139-2282 (1982).

4. M. Benderskii and L. Pastur, On the spectrum of the one dimensional Schrödinger equation with a random potential, Mat. Sb. 82:245-256 (1970).

5. J. Avron and B. Simon, Almost periodic Schrödinger operator II. The integrated density of states, Duke Math. J. 50:369-391 (1983).

6. T. Spencer, The Schrödinger equation with a random potential. A mathematical review, in Proceedings of the 1984 Les Houches Summer School, K. Osterwalder and R. Stora, eds. (North-Holland, New York, 1986).

7. L. Pastur, Spectral properties of disordered systems in one-body approximation, Commun. Math. Phys. 75:179 (1980).

8. F. Delyon and B. Souillard, Remark on the continuity of the density of states of ergodic finite difference operators, Commun. Math. Phys. 94:289 (1984).

9. W. Craig and B. Simon, Subharmonicity of the Lyaponov index, Duke Math. $J$. 50:551-560 (1983). 
10. E. Le Page, Empirical distribution of the eigenvalues of a Jacobi matrix, in Probability Measures on Groups VII (Lecture Notes in Mathematics, Vol. 1064; Springer, Berlin, 1983).

11. R. Carmona, A. Klein, and F. Martinelli, Anderson localization for Bernouli and other singular potentials, Commun. Math. Phys. 108:41-66 (1987).

12. B. Simon and M. Taylor, Harmonic analysis on $S L(2, \mathbf{R})$ and smoothness of the density of states in the one-dimensional Anderson model, Commun. Math. Phys. 101:1-19 (1985).

13. M. Campanino and A. Klein, A supersymmetric transfer matrix and differentiability of the density of states in the one-dimensional Anderson model, Commun. Math. Phys. 104:227-241 (1986).

14. P. March and A. Sznitman, Some connections between excursion theory and the discrete random Schrödinger equation with applications to analyticity and smoothness properties of the density of states in one dimension, Preprint.

15. A. Klein and A. Speis, Regularity of the invariant measure and of the density of states in the one dimensional Anderson model, J. Funct. Anal., to appear.

16. S. Edwards and D. Thouless, J. Phys. C 4:453 (1971).

17. F. Wegner, Bounds on the density of states in disordered systems, Z. Phys. B 44:9-15 (1981).

18. R. Maier, Bounds on the density of states of random Schrödinger operators, J. Stat. Phys. 48:425-448 (1987).

19. A. Bovier, M. Campanino, A. Klein, and F. Perez, Smoothness of the density of states in the Anderson model at high disorder, Commun. Math. Phys. 114: 439-461 (1988).

20. F. Constantinescu, J. Frohlich, and T. Spencer, Analyticity of the density of states and replica method for random Schrödinger operators on a lattice, J. Stat. Phys. 34:571-596 (1984).

21. A. Klein and A. Speis, Smoothness of the density of states in the Anderson model on a one-dimensional strip, Ann. Phys. (NY) 183:352-398 (1988).

22. C. Glaffig, Smoothness of the integrated density of states on discrete strip lattices, iid and non iid case, Preprint.

23. A. Klein, J. Lacroix, and A. Speis, Localization for the Anderson model on a strip with singular potentials, J. Funct. Anal., to appear.

24. P. Bougerol and J. Lacroix, Products of random matrices with applications to Schrödinger operators, in Progress in Probability and Statistics, Vol. 8 (Birkhauser, Boston, 1985).

25. K. B. Effetov, Supersymmetry and the theory of disordered metals, Adv. Phys. 32:53-727 (1983).

26. A. Klein and J. F. Perez, On the density of states for random potentials in the presence of a uniform magnetic field, Nucl. Phys. B 251 [FS13]:199-211 (1985).

27. A. Klein, L. J. Landau, and J. F. Perez, Supersymmetry and the Parisi-Sourlas dimensional reduction: A rigorous proof, Commun. Math. Phys. 93:459-482 (1984).

28. F. Martinelli and E. Scoppola, Introduction to the mathematical theory of Anderson localization, Nuovo Cimento 10 (10) (1987).

29. Ya. I. Gol'dsheid and A. G. Margulis, A condition for simplicity of the spectrum of Lyapunov exponents, Sov. Math. Dokl. 35:309-313 (1987).

30. T. Kato, Perturbation theory for linear operators (Springer-Verlag, 1980).

31. H. Kunz and B. Souillard, Sur le spectre des operateurs aux differences finies aleatoires, Commun. Math. Phys. 78:201-246 (1980).

32. H. Whitney, Trans. Amer. Math. Soc. 36:63 (1934). 